\title{
Comparing total hip arthroplasty and hemiarthroplasty in the treatment of hip fracture
}

\author{
Nicholas J. Tucker, Atul F. Kamath \\ Department of Orthopaedic Surgery, Orthopaedic and Rheumatology Institute, Cleveland Clinic, Cleveland, OH, USA \\ Correspondence to: Atul F. Kamath, MD. Department of Orthopaedic Surgery, Orthopaedic and Rheumatology Institute, Cleveland Clinic, 9500 \\ Euclid Ave, A41, Cleveland, OH 44195, USA. Email: kamatha@ccf.org. \\ Provenance: This is an invited article commissioned by the Section Editor Dr. Yu Zhang (Department of Sport Medicine and Adult Reconstructive \\ Surgery, Drum Tower Hospital, School of Medicine, Nanjing University, Nanjing, China). \\ Comment on: HEALTH Investigators, Bhandari M, Einhorn TA, et al. Total Hip Arthroplasty or Hemiarthroplasty for Hip Fracture. N Engl J Med \\ 2019;381:2199-208.
}

Submitted Nov 25, 2019. Accepted for publication Dec 06, 2019.

doi: $10.21037 /$ atm.2019.12.51

View this article at: http://dx.doi.org/10.21037/atm.2019.12.51

Many studies have been conducted to identify the preferred method of surgical intervention in the treatment of intracapsular hip fracture, specifically between the techniques of total hip arthroplasty (THA) and hip hemiarthroplasty (HHA). Considering that hip fractures are a major cause of death, disability, and high healthcare expenses throughout the world, research into ideal treatment selection has the potential for global impact. The authors of this editorial read with great interest the recent New England Fournal of Medicine publication on the Hip Fracture Evaluation with Alternatives of Total Hip Arthroplasty versus Hemi-Arthroplasty (HEALTH) trial.

While there is controversy and debate between the use of THA or HHA, the United Kingdom has developed clinical guidelines from their National Institute for Health and Care Excellence (NICE) that recommend THA over HHA in patients with a displaced intracapsular hip fracture who were able to walk independently out of doors with no more than the use of a stick, were not cognitively impaired, and were medically fit for anesthesia and the surgical procedure (1). Other countries have not established such explicit formal guidelines, but extensive research has been done to look at rates of serious adverse events, rates of complications, mortality, and patient functional scores after undergoing one of these two procedures.

This editorial comments on the HEALTH trial, an expertise-based randomized, controlled trial involving 1,495 patients (718 THA and $723 \mathrm{HHA}$ ) patients with a displaced femoral neck hip fracture (2). All patients were 50 years old or older with a low-energy displaced fracture of the femoral neck and had been able to ambulate without assistance before the fracture. The study focused on the need for a secondary hip procedure (reintervention) within 24 months, with additional analyses examining rates of serious adverse events, hip-related complications, death, and health-related quality of life and function.

The first major finding of the HEALTH study is that there is no identifiable difference regarding reintervention procedures between the THA and HHA groups after a 24-month follow-up $(\mathrm{P}=0.79)$. While this appears somewhat different from what some meta-analysis studies have reported (which show lower reoperation rates in the THA group), analysis of this study's Kaplan Meyer curves did indicate some significant advantage to THA over time (3-6). Although there was no significant difference between reintervention rates up to 12 months $(\mathrm{P}=0.32)$, there were less reinterventions in the THA group from 12 months to 24 months $(\mathrm{P}=0.01)$. Furthermore, the $\log$ of the hazard ratio between THA:HHA decreased by 0.097 each month $(\mathrm{P}=0.004)$. Despite no significant difference in rates of reintervention, when considering the post hoc analysis of reintervention hazard ratios and previous meta-analytic study results, THA may be preferred in functionally active cohorts.

Another major finding of the HEALTH study is that 
there are no significant differences between THA and HHA in the secondary end points of serious adverse events $(\mathrm{P}=0.13)$, hip-related complications ( $\mathrm{P}$ value not given), and mortality $(\mathrm{P}=0.48)$. One of the major pieces of evidence used by the paper in its discussion section to suggest that HHA could be preferred over THA is the claim that patients in the THA group had more serious adverse events and more cases of hip instability or dislocation. While results have been mixed about this in other studies, neither of these findings were found to be significant in this study. Furthermore, the hazard ratio confidence intervals (nonsignificant) for these secondary end points cannot be used as evidence to suggest that one of these procedures was more likely to the particular end point.

The study's lack of a significant difference in mortality is also different from existing studies in the literature. For example, a study using 5,590 propensity-matched patients demonstrated THA patients to have a reduced risk for mortality when compared to HHA patients $(\mathrm{P}=0.029)$ and even to have a decreased risk of 30-day hospital stays $(\mathrm{P}=0.017)$ (7). Another study found the one-year costs for THA patients to be as much as Can $\$ 2,700$ lower than the costs for HHA patients (8). These two papers support THA having both a potential survival and economic benefit which is becoming ever more important with the increased focus on value-based care.

When there actually was a significant difference found in the WOMAC (Western Ontario and McMaster Universities Osteoarthritis Index) assessment scores for pain, stiffness, and function, with THA outperforming HHA in all measures, the WOMAC differences did not meet thresholds of minimal clinically important difference (MCID) $(9,10)$, despite some variability in published MCID thresholds (11). In addition, one of the noted drawbacks from the study design was that it was unblinded in the assessment of function, leaving open the possibility for bias when assessing function. Support from other randomized, controlled trials still seems to attribute the advantages of improved function and quality of life to the THA group $(12,13)$.

Though this paper's randomized, controlled trial design and large sample size are impressive, some obvious limitations are the $14.9 \%$ of patients lost to follow-up and 75 patients who were excluded from analysis because they did not receive the procedure to which they were assigned. While demographics of patients do not seem to have differed between these groups, there is a substantial amount of acknowledged missing data as well.

In summary, the HEALTH study ultimately reports no clinically significant difference between THA and HHA in rate of reintervention at 24 months, serious adverse events, hip-related complications, mortality, or functionality. The only differences found were that THA has a lower risk of reintervention in the 12-24 months period, and the THA group had superior yet sub-MCID threshold WOMAC scores. Based on much of the comparative literature, including a moderate strength rating from the American Academy of Orthopaedic Surgeons, THA has generally been believed to be the most beneficial for physiologically younger and more active patients $(4,5,14)$. This belief among many orthopaedic surgeons is thought to be based on the exclusion criteria of many of the randomized controlled trials that have demonstrated a functional advantage to THA $(12,13)$. The individual surgeon bias may also relate to training and experience, as well as potential subspecialty fellowship training (e.g., adult lower extremity reconstruction/ arthroplasty, trauma, sports). To examine these questions more closely, future studies may benefit from expanding inclusion criteria and conducting subanalyses within the randomly assigned THA and HHA groups to see if there are any demonstrable differences among patients with differing ages, co-morbidities, or pre-surgery activity levels. Overall, while the HEALTH trial certainly explores the debate between THA and HHA, it may not provide any compelling reason to alter current NICE or AAOS guidelines without more evidence, particularly when many other studies have found better functional outcomes in patients undergoing THA for intracapsular hip fractures.

\section{Acknowledgments}

None.

\section{Footnote}

Conflicts of Interest: The authors have no conflicts of interest to declare.

Ethical Statement: The authors are accountable for all aspects of the work in ensuring that questions related to the accuracy or integrity of any part of the work are appropriately investigated and resolved.

\section{References}

1. National Institute for Health and Care Excellence. Hip 
Fracture: management (NICE Guideline CG124). 2011. Available online: https://www.nice.org.uk/guidance/cg124

2. HEALTH Investigators, Bhandari M, Einhorn TA, et al. Total Hip Arthroplasty or Hemiarthroplasty for Hip Fracture. N Engl J Med 2019;381:2199-208.

3. Hopley C, Stengel D, Ekkernkamp A, et al. Primary total hip arthroplasty versus hemiarthroplasty for displaced intracapsular hip fractures in older patients: systematic review. BMJ 2010;340:c2332.

4. Burgers PT, Van Geene AR, Van den Bekerom MP, et al. Total hip arthroplasty versus hemiarthroplasty for displaced femoral neck fractures in the healthy elderly: a meta-analysis and systematic review of randomized trials. Int Orthop 2012;36:1549-60.

5. Lewis DP, Waever D, Thorninger R, et al. Hemiarthroplasty vs total hip arthroplasty for the management of displaced neck of femur fractures: a systematic review and meta-analysis. J Arthroplasty 2019;34:1837-1843.e2.

6. Yu L, Wang Y, Chen J. Total hip arthroplasty versus hemiarthroplasty for displaced femoral neck fractures: Meta-analysis of randomized trials hip. Clin Orthop 2012;470:2235-43.

7. Warren JA, Sundaram K, Anis HK, et al. Total Hip Arthroplasty Outperforms Hemiarthroplasty in Patients Aged 65 Years and Older: A Propensity-Matched Study of Short-Term Outcomes. Geriatr Orthop Surg Rehabil 2019;10:2151459319876854.

8. Ravi B, Pincus D, Khan H, et al. Comparing complications and costs of total hip arthroplasty and hemiarthroplasty

Cite this article as: Tucker NJ, Kamath AF. Comparing total hip arthroplasty and hemiarthroplasty in the treatment of hip fracture. Ann Transl Med 2019;7(Suppl 8):S259. doi: 10.21037/ atm.2019.12.51 for femoral neck fractures. J Bone Joint Surg Am 2019;101:572-9.

9. Tubach F, Ravaud P, Baron G, et al. Evaluation of clinically relevant changes in patient reported outcomes in knee and hip osteoarthritis: the minimal clinically important improvement. Ann Rheum Dis 2005;64:29-33.

10. Impellizzeri FM, Mannion AF, Naal FD, et al. The early outcome of surgical treatment for femoroacetabular impingement: success depends on how you measure it. Osteoarthritis Cartilage 2012;20:638-45.

11. MacKay C, Clements N, Wong R, et al. A systematic review of estimates of the minimal clinically important difference and patient acceptable symptom state of the Western Ontario and McMaster Universities Osteoarthritis Index in patients who underwent total hip and total knee replacement. Osteoarthritis Cartilage 2019;27:1408-19.

12. Macaulay W, Nellans KW, Garvin KL, et al. Prospective Randomized Clinical Trial Comparing Hemiarthroplasty to Total Hip Arthroplasty in the Treatment of Displaced Femoral Neck Fractures: Winner of the Dorr Award. J Arthroplasty 2008;23:2-8.

13. Hedbeck CJ, Enocson A, Lapidus G, et al. Comparison of bipolar hemiarthroplasty with total hip arthroplasty for displaced femoral neck fractures: a concise four-year follow-up of a randomized trial. J Bone Joint Surg Am 2011;93:445-50.

14. American Academy of Orthopedic Surgeons. Management of hip fractures in the elderly: summary. 2019. Available online: https://www.aaos.org/cc_files/aaosorg/research/ guidelines/hipfxsummaryofrecommendations.pdf 